ARTICULO INVITADO

\title{
Bases de las Estrategias de Control Químico del Tizón Tardío de la Papa Desarrolladas por PROINPA en Bolivia
}

\author{
Enrique N. Fernández-Northcote, Oscar Navia, \\ Antonio Gandarillas ${ }^{1}$
}

\begin{abstract}
Resumen
El tizón de la papa causado por Phytophthora infestans es una de las enfermedades más importantes que afecta mundialmente a este cultivo. En países desarrollados la enfermedad es controlada con relativa facilidad mediante el empleo de fungicidas, pero en Bolivia como en otros países en desarrollo el alto costo de los fungicidas limita su utilización. Esto es agravado por el desconocimiento de estrategias de aplicación de fungicidas y su uso racional. Experimentos realizados por PROINPA durante varias campañas agrícolas entre los años 1992-1997 en zonas "tizoneras" de Bolivia sobre estrategias para el control químico, han permitido determinar y confirmar estrategias efectivas, eficientes, económicas, y sensibles con el medio ambiente y la salud humana, para el control del tizón tanto en cultivares susceptibles como en resistentes. La aplicación de estas estrategias por los agricultores está permitiendo que se utilicen cultivares susceptibles, pero localmente valiosos, en zonas y épocas donde ya no se les podía cultivar, así como interesarse nuevamente en cultivares que en estas mismas zonas consideraban habían perdido su resistencia. En el presente artículo se presentan las bases técnicas de dichas estrategias, las que consideran su necesidad, los tipos de fungicidas disponibles en el mercado y sus características, la oportunidad apropiada para su uso tomando en cuenta su interacción con el hospedante y su acción en las fases del ciclo biológico de $P$. infestans, y la idiosincrasia del agricultor andino de aquellas zonas en las que esta enfermedad los ha llevado a una cultura de "evasión" del problema, a una agricultura de subsistencia, y frecuentemente a la emigración de las zonas afectadas.
\end{abstract}

1 Ph.D. Consultor (Ex - Jefe Dpto. Fitopatología), Ing. Agr. Investigador y Ph.D. Director, respectivamente. Fitopatólogos, PROINPA (Fundación para la Promoción e Investigación de Productos Andinos), Casilla 4285, Cochabamba, Bolivia. 
Aceptado para publicación: junio 1999.

Palabras claves adicionales: Phytophthora infestans, tizón tardío, integración resistencia - control químico, manejo integrado.

\title{
Basis of the Strategies for the Chemical Control of Potato Late Blight Developed By PROINPA in Bolivia
}

\begin{abstract}
Summary
Potato late blight caused by Phytophthora infestans is one of the most important diseases of the potato crop in Bolivia and the rest of the world as well. In developed countries the disease is relatively easily controlled by the use of fungicides, but in Bolivia, as in other developing countries, the high cost of fungicides limits their use. Control of the disease is made more difficult by the lack of knowledge of good fungicide use and strategies for chemical control. Field experiments with strategies for chemical control of late blight in several crop seasons and potato late blight areas of Bolivia carried out by PROINPA between 1992 and 1997 have made it possible to identify and validate strategies for both susceptible and resistant potato cultivars. These strategies are effective, efficient, economic, and better for human health and the environment, than existing practices. By using these strategies, farmers can grow again susceptible, but locally valuable potato cultivars in areas and growing seasons regularly affected by late blight and they are also interested again in using cultivars whose resistance had previously been considered as lost. This paper presents the technical basis of the strategies, considering the need for them, the kinds and characteristics of fungicides available in the market, the most appropriate moment for their use taking into account the hostpathogen interaction and the plant growth cycle, and the idiosyncrasy of the Bolivian Andean farmers in areas affected by late blight where the disease has created a culture of disease "evasion", enhanced farming for subsistence and increased the out migration.
\end{abstract}

Additional index words:

Phytophthora infestans, integration of resistance - chemical control, integrated pest management. 


\section{Introducción}

El tizón de la papa causado por Phytophthora infestans (Mont.) de Bary es una de las enfermedades más importantes que afecta al cultivo de la papa (Solanum tuberosum L). En varias oportunidades la enfermedad ha alcanzado mayores proporciones y efectos desastrosos. La más documentada es la hambruna que ocasionó en Irlanda en 1845 cuando prácticamente la mitad del cultivo de papa fue destruido y al año siguiente causó la pérdida total de la producción. Cerca de un millón de personas murió como consecuencia de la hambruna y otro millón tuvo que emigrar hacia otras regiones.

No obstante la gran cantidad de conocimiento existente sobre la enfermedad, el tizón continúa siendo uno de los principales factores limitantes de la producción de papa en el mundo. Si no es controlado las pérdidas pueden llegar al 100\%, e incluso, con niveles bajos de infección, la cosecha puede resultar no apta para el almacenamiento.

El tizón de la papa afecta en Bolivia aproximadamente 20,000 ha del cultivo, de las cuales una gran parte está dedicada a la producción de semilla, incluyendo la del sector formal, de los cultivares Waycha, Sani Imilla, Alpha y Desirée. En la Provincia Ayopaya del Departamento de Cochabamba, donde PROINPA ha realizado la mayoría de sus investigaciones sobre el tizón a nivel de campo, se cultivan alrededor de 5,600 ha de papa anualmente. Para los agricultores de esta zona, el tizón es el principal factor limitante, favorecido por una precipitación relativamente alta (alrededor de 1,000 $\mathrm{mm}$ año) y temperaturas medias de $11-25{ }^{\circ} \mathrm{C}$ (mínima -máxima). En las zonas bajas con mayor presión de tizón algunos agricultores utilizan cultivares mejorados resistentes como Puka Toralapa (CIP 720085) o Runa Toralapa (Perricholi $=$ CIP 374080.5). En las zonas más altas de Ayopaya, la gran mayoría de los agricultores siembran el cultivar susceptible Waycha (S. tuberosum ssp. andigena).

Los agricultores generalmente empiezan las aplicaciones de fungicidas solamente cuando ven síntomas de la enfermedad. Tradicionalmente aplican con mochilas y usan dosis en un $30 \%$ por debajo de lo recomendado, fumigan de 2 a 4 veces, dejan intervalos largos entre aplicaciones (Salazar, 1999; Tórrez y Thiele, 1998) y no realizan una buena cobertura de las plantas asperjadas. Bajo estas condiciones de aplicación de fungicidas, en años con poca incidencia, los cuales son excepcionales, los agricultores logran cosechas razonables; sin embargo, normalmente el tizón es severo, causando la pérdida de la mayor parte de la producción potencial. Es común ver parcelas 
abandonadas donde el agricultor no ha logrado controlar la enfermedad. Las pocas instituciones, pero las más importantes a nivel nacional dedicadas a la producción de semilla en esta Provincia y en la de Carrasco, también del Departamento de Cochabamba, normalmente realizan entre 11 y 16 aplicaciones para poder lograr una buena producción de tubérculos-semilla.

En países desarrollados, la enfermedad es controlada con relativa facilidad mediante el empleo de fungicidas, pero en Bolivia como en otros países en desarrollo, el alto costo de los mismos limita su utilización. Esto es agravado por factores climáticos muy favorables para el desarrollo de la enfermedad y continuidad del cultivo, desconocimiento de estrategias de aplicación de fungicidas apropiadas para el tizón, uso irracional de los fungicidas, y desconocimiento de los beneficios socioeconómicos que se logran con un buen control químico de esta enfermedad.

Con el fin de evitar o disminuir las pérdidas ocasionadas por esta enfermedad, es necesario implementar el manejo integrado del tizón (Fernández-Northcote y Navia, 1995; Navia y Fernández-Northcote, 1996, 1999). Entre sus componentes, el control químico sigue siendo el componente más importante. Desafortunadamente, no solamente en los países en vías de desarrollo pero también en los desarrollados, el control químico se está haciendo más difícil debido a la aparición de poblaciones nuevas y más agresivas de $P$. infestans y que aparentemente se están creando con la diseminación del tipo de apareamiento sexual A2 (Fry et al., 1993; Flier et al.,1998). Por otro lado, el control químico tiene que ser muy eficiente y compatible con la tendencia contemporánea de reducir su uso al mínimo para evitar su impacto en el medio ambiente y en la salud humana.

Experimentos sobre estrategias de control químico del tizón realizados por PROINPA durante varias campañas agrícolas entre los años 1992 y 1997, en zonas "tizoneras" de Bolivia, han permitido determinar y confirmar estrategias efectivas, eficientes, económicas, y sensibles con el medio ambiente y la salud humana, para el control químico del tizón tanto en cultivares susceptibles como en su integración con cultivares resistentes (Fernández-Northcote et al. Datos no publicados; Navia et al., 1996; Navia y Fernández-Northcote, 1996); Rojas, 1995; Velasco, et al., 1996). Estos trabajos se han realizado en parcelas de agricultores ubicadas entre los $2100 \mathrm{msnm}$ (Zonas de Valle Bajo) y los 3400 msnm (Zonas de Altura) con características climáticas durante el desarrollo del cultivo, 
caracterizadas por una precipitación pluvial entre los 650 y $1713 \mathrm{~mm}$ y temperaturas medias de $11^{\circ} \mathrm{C}$ mínima y $25^{\circ} \mathrm{C}$ máxima. En el presente artículo se presentan las bases técnicas de dichas estrategias.

\section{Necesidad de una estrategia de control químico}

La papa es un cultivo básico y de seguridad alimentaria para la población de Bolivia. Para más de 200,000 familias de pequeños agricultores que constituyen entre el 30 al $40 \%$ del total de campesinos del país (Zeballos,1997), la papa constituye la principal fuente de alimentación e ingresos. En su gran mayoría, los agricultores son de bajos recursos económicos y la superficie que utilizan no es mayor de una hectárea. El consumo per cápita es entre 100 a 120 kg/año (Zeballos,1997), siendo éste uno de los más altos del mundo.

Cerca de 130,000 ha son dedicadas al cultivo de la papa, con un rendimiento promedio en los Andes Bolivianos de $5 \mathrm{t} / \mathrm{ha}$, mientras que el mundial es de 14 t/ha y de 26 t/ha en los países desarrollados (Horton, 1992; Zeballos, 1997). En las 20,000 ha de papa afectadas por el tizón la enfermedad ocasiona al presente una pérdida directa de alrededor de US\$ 30 millones/año y de US\$ 100 millones/año en rendimiento potencial. La mayor parte del área afectada está en regiones productoras de semilla que actualmente escasamente cubren el $1 \%$ de las necesidades urgentes nacionales de semilla de calidad. Esta pérdida indirecta que ocasiona la enfermedad, magnifica la importancia del tizón en Bolivia como un factor limitante en la producción y productividad del cultivo de la papa, el cultivo más importante en la economía agrícola del país (Bojanic, 1955).

Como consecuencia del tizón y la falta de conocimientos para una estrategia de control químico, el agricultor ha desarrollado una cultura de evasión de la enfermedad ya sea en el tiempo, mediante la utilización de otras épocas de siembra en que no se presenta la enfermedad o lo hace con más irregularidad, o en el espacio, cultivando en las épocas de mayor incidencia del tizón, pero sembrando en las partes de mayor altitud (más de $3400 \mathrm{msnm}$ ) donde las temperaturas más bajas no son favorables al desarrollo de la enfermedad.

La cultura de evasión de la enfermedad implica una baja productividad del área bajo cultivo que no concuerda con la preocupación contemporánea de incrementar la productividad 
por razones medioambientales y de la necesidad alimentaria presente y futura. Se prevé que en el término de los próximos 20 a 30 años la producción mundial de alimentos necesaria deberá ser el doble de lo que se necesita al presente. Por lo tanto es conveniente desarrollar e implementar y capacitar al agricultor en estrategias para el control químico del tizón que le permitan incrementar la productividad del cultivo de la papa combatiendo eficientemente la enfermedad aún bajo condiciones más favorables para su desarrollo.

\section{Tipos de fungicidas utilizados para el control químico del tizón}

Dos tipos de fungicidas son utilizados para el control químico del tizón, los de contacto (más propiamente no sistémicos, llamados también residuales) y los sistémicos.

Los de contacto son comúnmente referidos también como fungicidas protectores o preventivos y los sistémicos como curativos. En opinión de los autores estos términos no están bien aplicados y por otro lado confunden al agricultor. Así, éstos aplican los fungicidas sistémicos sólo cuando ven los síntomas del tizón (porque los consideran "curativos"), lo cual es normalmente tarde para un buen control de la enfermedad. Por lo tanto en el presente artículo utilizaremos los términos de contacto y sistémicos.

Los fungicidas de contacto afectan las estructuras del patógeno en la superficie de la planta actuando en sus fases de germinación y penetración. Una vez que el patógeno ha entrado en la planta estos fungicidas no lo afectan. Como los brotes nuevos y las partes de la planta que desarrollan luego de una aplicación deben ser protegidos y si lloviera luego de la aplicación, ésta debería repetirse; un buen control del tizón con este tipo de fungicidas sólo se logra con aplicaciones frecuentes y a intervalos cortos entre las aplicaciones lo que lleva a tener que realizar entre 16 a 20 aplicaciones en zonas propicias para el tizón. Aún bajo estas condiciones, el control podría no ser muy efectivo si es que las condiciones son muy favorables para la enfermedad y si es que las condiciones lluviosas no dejara realizar las aplicaciones en el momento oportuno.

Con los fungicidas de contacto es muy importante mantener una capa apropiada del fungicida en el follaje, tanto en el haz 
como en el envés de la hoja. Se necesita cubrir toda la parte aérea de la planta.

Su acción será efectiva siempre que persistan en la hoja y no sean lavados por la lluvia, es decir tengan buena tenacidad.

Las características y condiciones para la efectividad de los fungicidas de contacto, anteriormente mencionadas, además del hecho de que comúnmente el agricultor no realiza las aplicaciones debidamente por cuanto no cubre bien la planta, por desconocimiento de esta necesidad y por falta de tiempo, la eficacia de la acción de estos fungicidas es afectada en forma significativa. El desarrollo de los fungicidas sistémicos a partir de 1970 abrió una alternativa para solucionar estos problemas y llegar a un mejor control del tizón.

Los fungicidas sistémicos (Tabla 1) penetran en la planta y se movilizan translaminarmente del haz al envés o viceversa y luego del punto donde cayeron hacia la parte superior de la planta, es decir tienen un movimiento acropétalo. Sólo uno de los fungicidas sistémicos que se utilizan para el control del tizón y que no es propiamente un fungicida, el fosetil-aluminio, se moviliza también en dirección basipétala, es decir de donde penetra hacia la parte inferior de la planta. En el caso de los fungicidas sistémicos una aplicación constante y uniforme no es tan importante como en los fungicidas de contacto. Luego de su aplicación el fungicida sistémico penetra en la planta y se moviliza acropetalamente aún hacia partes de la planta no alcanzadas por la aplicación. El intervalo entre aplicaciones puede ser distanciado y el fungicida no es lavado por las lluvias poco después de su aplicación. Estas son las grandes ventajas de los fungicidas sistémicos sobre los fungicidas de contacto especialmente en las zonas muy favorables al tizón. Las desventajas de los fungicidas sistémicos son su mayor costo y la selección e incremento de resistencia en el patógeno cuando se les utiliza inapropiadamente.

Para manejar el problema de la resistencia a los fungicidas sistémicos, el Grupo Internacional de Asociaciones Nacionales de Fabricantes de Agroquímicos ha constituido el comité de acción sobre resistencia a fungicidas (FRAC) que ha propuesto recomendaciones generales diseñadas para prevenir 0 demorar el problema de la resistencia. Entre éstas, una de las más importantes es la comercialización de los fungicidas sistémicos en mezclas con fungicidas de contacto.

\section{Fungicidas de contacto:}


Cúpricos. Los fungicidas cúpricos fueron los primeros en ser utilizados para el control del tizón. Los más utilizados son el caldo Bórdales y en tiempos recientes los fungicidas a base de oxicloruro de cobre y de óxido cuproso. Entre los fungicidas de contacto los cúpricos tienen la ventaja de tener una buena tenacidad. Como desventaja tienden a retrasar el desarrollo vegetativo de la planta y por ello se recomienda su utilización después de empezada la floración. Tienen acción en los esporangios y las zoosporas del patógeno en las que denaturalizan enzimas de la cadena respiratoria (Schwinn and Margot, 1991).

La tendencia moderna para mejorar la efectividad de los fungicidas cúpricos ha estado orientada a reducir el tamaño de sus partículas y cristales, así como permitir que sean absorbidos por el follaje y tornarse sistémicos.

Ditiocarbamatos. Los ditiocarbamatos son compuestos orgánicos azufrados derivados del ácido bisditiocarbámico. Sus sales vienen a ser 1,2-bisditiocarbamatos estando el nombre químico del fungicida relacionado al grupo químico (metil, etil, etc.) y al metal involucrado. Los ditiocarbamatos más utilizados en el control del tizón son el zineb (etileno y zinc), maneb (etileno y manganeso), mancozeb (etileno, zinc y manganeso), metiram (etileno y zinc) y propineb (propileno y zinc).

A diferencia de los cúpricos, los ditiocarbamatos no son fitotóxicos por lo que pueden ser utilizados en cualquier etapa del desarrollo de la planta. Sin embargo, tienen la desventaja de tener baja tenacidad es decir son fácilmente lavados por la lluvia. Su acción es principalmente durante la germinación de los esporangios y zoosporas asi como en el desarrollo micelial inactivando aminoácidos o procesos bioquímicos importantes que involucran enzimas con grupos tioles (Schwinn and Margot, 1991).

Los ditiocarbamatos son los fungicidas más utilizados actualmente en el control del tizón. Aunque el bajo costo facilita su utilización, las estrategias de control basadas en gran parte o exclusivamente en el uso de ellos, no son convenientes en zonas de alta incidencia (muy tizoneras) debido a la necesidad de hacer aplicaciones frecuentes. La mayor frecuencia de aplicación incrementa la exposición del agricultor y del medio ambiente. En Suecia el uso de maneb y propineb han sido suspendidos por razones relacionadas con la salud humana y/o el medio ambiente (Emmerman, 1996). En otros países como Dinamarca, se han implementado políticas para reemplazar al maneb (Jorgensen, 1996). La EPA de los Estados Unidos de 
Norte América tiene evidencias suficientes por estudios realizados con animales de un probable efecto cancerígeno del maneb, zineb, y mancozeb en humanos (The Pesticides Trust, 1994). Crissman et al. (1994) y Cole et al. (1997) han reportado casos de dermatitis crónica asociada al empleo frequente de mancozeb en el Ecuador.

Tabla 1. Fungicidas sistémicos y su tipo de movimiento en la planta de papa

\begin{tabular}{lrrr}
\hline $\begin{array}{l}\text { Nombre común del } \\
\text { producto químico }\end{array}$ & $\begin{array}{c}\text { Penetración } \\
\text { translaminar }\end{array}$ & $\begin{array}{c}\text { Movimiento } \\
\text { acropétalo }\end{array}$ & $\begin{array}{c}\text { Movimiento } \\
\text { basipétalo }\end{array}$ \\
Cimoxanil & ++ & + & - \\
Propamocarb & ++ & $(+)$ & - \\
Dimetomorf & ++ & $(+)$ & - \\
Fenilamidas & ++ & ++ & $(+)$ \\
Fosetil-aluminio & ++ & ++ & ++ \\
\hline
\end{tabular}

++=marcado,rápido; +=débil,lento (+)= mínimo; -= no hay.

Phtalimidas. Folpet, captafol, y captan pertenecen a este grupo de fungicidas. Los dos primeros han sido importantes en el control del tizón. Captafol ha sido considerado por WHO (The World Health Organization) como extremadamente peligroso (The Pesticides Trust, 1994). En la actualidad se está promoviendo el uso de captan. Su acción fungicida y fungistática contra las estructuras fungosas es sólo en la superficie de las hojas. Afectan la movilidad de las zoosporas y su germinación. Reaccionan con grupos tioles que llevan a una serie de efectos bioquímicos no específicos que afectan la actividad enzimática (Schwinn and Margot, 1991).

Phtalonitrilos. En este grupo se encuentra el clorotalonil cuya diferencia con los ditiocarbamatos y phtalimidas está en su mayor tenacidad y redistribución, adhiriéndose con firmeza en la superficie del follaje y permitiendo aplicaciones a intervalos mayores que los necesarios con los fungicidas mencionados. Su acción en las células fungosas es atrapando el glutation libre, previniendo la activación (reducción) de la gliceroaldehido-3-fosfato dehidrogenasa y quizás otras enzimas similares (ISK-Biotech, 1990).

Piridineaminas. Fluazinam es un fungicida nuevo perteneciente a este grupo. Se le atribuye buen potencial por su mayor tenacidad en la superficie de las hojas con relación a otros fungicidas de contacto. Su empleo en concentraciones 
bajas permite reducir la cantidad de fungicida aplicado por hectárea. Inhibe la germinación de esporas, la formación de apresorios, la penetración y el crecimiento de hitas, así como la esporulación. Fluazinam es un desacoplador potente en la fosforilación oxidativa e inhibe la transferencia de protones a través de la membrana mitocondrial (Guo et al., 1990).

Estáñicos. Los compuestos trifenilestaño acetato y trifenilestaño hidróxido son efectivos para el control del tizón, poseen una tenacidad superior a la de los cúpricos y ditiocarbamatos. Actúan sobre las esporas e hifas afectando la fosforilación oxidativa (Schwinn and Margot, 1991). Sin embargo, la fitotoxicidad de estos fungicidas limita su utilización hacia el final en el desarrollo del cultivo. Estos fungicidas figuran en listas de la Comunidad Europea para ser considerados como productos químicos cuyo uso debe prohibirse o reducirse con el propósito de proteger el medio ambiente acuático (The Pesticides Trust, 1994).

\section{Fungicidas sistémicos:}

Cianoacetamida-Oximas. Cimoxanil pertenece a este grupo de fungicidas. Su movimiento en la planta es sólo translaminar, no tiene movimiento acropétalo. Su persistencia en la planta está limitada a unos pocos días por lo que los intervalos para su aplicación apropiada es similar a la de los fungicidas de contacto. Cimoxanil afecta la síntesis de ADN y en menor grado la de ARN (Ziogas et al.,1987). Se ha reportado un efecto sinergístico cuando se usa cimoxanil en mezcla con mancozeb. La mezcla controla mejor el tizón que cuando se usa cimoxanil o mancozeb en forma independiente (Samoucha y Cohén, 1989; Evenhuis et al., 1996).

Carbamatos. Propamocarb $\mathrm{HCl}$ pertenece a este grupo. Es un fungicida básicamente translaminar. Tiene poca acción en la germinación del esporangio. Actúa sobre el micelio joven afectando la permeabilidad de la membrana celular, pero no es efectivo cuando el micelio ya ha desarrollado (Papavizas et al., 1978). Propamocarb no es efectivo cuando se le aplica en plantas ya infectadas, y no ejerce control cuando estas ya tienen tres días de infectadas (Samoucha y Cohén, 1990) por lo que para actuar de forma efectiva el intervalo entre sus aplicaciones debe ser corto (siete días).

Derivados del ácido cinámico. El dimetomorf es uno de los fungicidas más recientes. Se le comercializa desde 1990. Es básicamente un fungicida translaminar reportándosele una 
excelente actividad antiesporulante que previene la producción de esporangios y oosporas. El dimetomorf afecta la formación de la pared celular promoviendo su lisis y la muerte de la célula. Se le considera de bajo riesgo como promotor del desarrollo de resistencia en el patógeno (Leroux et al., 1993).

Fenilamídas. Metalaxil fue el primer fungicida de este grupo introducido al mercado en 1977. Posteriormente otras tres fenilamidas se han venido comercializando: ofurace, benalaxil, y oxadixil. Estos fungicidas crearon una gran expectativa por su acción post-infeccional y actividad sobresaliente bajo condiciones muy favorables para la enfermedad. El uso extensivo e intensivo de metalaxil como único fungicida en el control del tizón a lo largo de la campaña y debido a su frecuente utilización cuando ya se observaban síntomas de la enfermedad, creó una presión para la aparición de variantes de $P$. infestans resistentes al metalaxil a los tres años de su introducción (Davidse et al., 1981; Dowley y O'Sullivan, 1981). Su comercialización posterior en mezclas con fungicidas de contacto y siguiendo recomendaciones del FRAC (Fungicide Resistance Action Commitee, o Comité de Acción en Resistencia a Fungicidas)(Wade y Delp, 1985) ha permitido la utilización del metalaxil y otras fenilamidas para el control efectivo, eficiente y estable del tizón, a pesar de la presencia al inicio de la campaña de 30 a $50 \%$ de variantes de P.infestans resistentes a fenilamidas (Nuninger et al., 1995; Dowley et al., 1995). Diferentes estudios han mostrado que aunque la frecuencia de las variantes resistentes a fenilamidas aumente durante una campaña en la magnitud de la presión que se ejerza para su selección, la frecuencia disminuye al inicio de la siguiente campaña debido probablemente a una menor capacidad de supervivencia en comparación con las variantes no resistentes a las fenilamidas (Williams y Gisi, 1992).

Metalaxil tiene poco efecto en la germinación del esporangio o de las zoosporas o en la movilidad de las zoosporas. La penetración y la formación de haustorios en la planta de papa no son afectadas. Metalaxil ejerce su efecto fungitóxico sólo en el interior de la planta (Staub et al., 1980, citado en Schwinn y Margot, 1991). Las fenilamidas inhiben severamente la esporulación y en grado menor el desarrollo del micelio (Bruck et al., 1980). Las fenilamidas interfieren en la síntesis de ARN inhibiendo a la enzima ARN polimerasa. La mejor redistribución y la mayor persistencia de las fenilamidas en las plantas se logra en las plantas jóvenes en crecimiento activo. Entre los pesticidas evaluados por la EPA oxadixil figura como posible cancerígeno en humanos en base a información muy limitada 
obtenida en animales (The Pesticides Trust, 1994).

Fosfitos. El fosetil-aluminio pertenece a este grupo. Tiene una acción directa propiamente como la de un fungicida, pero además actúa indirectamente activando mecanismos de defensa propios de la planta. Otra ventaja de este compuesto es su movilización en la planta tanto acropetalamente como basipetalamente, por lo que con este producto se puede lograr un buen control de la infección en tubérculos. El fosetil-aluminio es un compuesto que penetra rápidamente en la planta y tiene una larga persistencia (algunas semanas), lo que permite alargar el intervalo entre aplicaciones.

\section{Oportunidad de aplicación de los fungicidas}

En base a lo descrito anteriormente para los diferentes fungicidas que se emplean en el control del tizón, en la Figura 1 se muestra su acción con relación a las diferentes fases del ciclo biológico de $P$. infestans. Los fungicidas de contacto actúan sólo en las primeras horas del primer contacto del esporangio con la superficie húmeda de la hoja o de otra parte aérea de la planta de papa, en las fases de esporangio, liberación de zoosporas, germinación de zoosporas, formación del tubo germinativo, formación de apresorios, y penetración inicial. Los fungicidas cimoxanil, propamocarb y fosetil-al también actúan en estas fases. Una vez que $P$. infestans ha penetrado en la planta actúan los translaminares en la parte de la planta donde fueron depositados por la aplicación, y los sistémicos acropétalos y basipétalos donde lograron movilizarse luego de su aplicación y penetración en la planta. Cuanto más joven, hidratada y vigorososa es la planta, la movilización de los sistémicos será mejor así como su persistencia de acción. Por ello no es aconsejable su utilización en fases tardías en el desarrollo del cultivo, aparte de que se ha observado un efecto estimulante del desarrollo vegetativo por los sistémicos acropétalos con muy buen control de la enfermedad, pero en perjuicio de una mayor producción. Los fungicidas de contacto, cimoxanil, y fosetil-al actúan también sobre los esporangióforos y esporangios que salen a través de los estomas generalmente en la cara inferior de los foliolos. En base a estas características y otras descritas anteriormente los períodos principales de uso de fungicidas para el control del tizón se ilustran en la Figura 2. 
Fig. 1. Acción de los fungicidas empleados para el control del tizón en fases del ciclo biológico de Phytophthora infestans en el foliolo de papa de un cultivar susceptible. A Se indican las estructuras del patógeno. B Se indican las estructuras del foliolo del hospedante y la manifestación de necrosis en el foliolo. 


\section{Estrategias de PROINPA para el control químico del tizón de la papa}

Con la información y experiencias anteriores, PROINPA desarrolló las estrategias para el control químico del tizón en cultivares susceptibles actualmente los más cultivados en Bolivia, así como para cultivares resistentes disponibles al presente y que vienen utilizando los agricultores en menor escala. En ciertas zonas muy tizoneras bajo el manejo del agricultor estos cultivares resistentes se comportan como susceptibles, sin embargo son los únicos que pueden cultivar. Por otro lado, PROINPA está liberando cultivares resistentes los que deben ser utilizados con una estrategia apropiada de control químico para su mejor manejo y durabilidad.

Las estrategias para cultivares susceptibles están basadas en:

- la aplicación en forma preventiva de fungicidas 10 días después del $80 \%$ de emergencia, es decir, antes de que aparezca el tizón,

- la alternancia del uso de fungicidas sistémicos y de contacto,

- la no utilización de fungicidas sistémicos en más de tres oportunidades, durante la campaña,

- frecuencias de aplicación de 7 a 14 días según las condiciones climáticas muy favorables a poco favorables, respectivamente.

En una zona de clima muy propicio para el desarrollo del tizón (zona muy tizonera), se recomienda que las aplicaciones comiencen con un fungicida sistémico (Estrategia 1, Figura 3). En estas zonas el tizón suele presentarse desde muy temprano en la campaña debido a que la temperatura $\left(11-25^{\circ} \mathrm{C}\right)$ y especialmente la precipitación pluvial son muy favorables para su desarrollo. La precipitación total durante la campaña está entre 800 y $1700 \mathrm{~mm}$. En una zona de clima no muy tizonero, las aplicaciones pueden comenzar con un fungicida de contacto (Estrategia 2, Figura 3). En estas zonas la aparición del tizón desde muy temprano en la campaña no es frecuente debido a condiciones de temperatura mínima inferior a $11^{\circ} \mathrm{C}$ y/o precipitación semanal acumulada inferior a $30 \mathrm{~mm}$. El momento del comienzo de la enfermedad es variable. La precipitación total en la campaña es generalmente inferior a $800 \mathrm{~mm}$. 
La estrategia para cultivares resistentes (Figura 4) se basa en lo siguiente:

- la aplicación de un fungicida sistémico cuando se observan los primeros síntomas del tizón,

- continuar con la aplicación de un fungicida de contacto cuando se observe que la enfermedad continúa su avance, es decir que el patógeno esté esporulando y/o se observe que la sintomatología esté progresando,

- continuar aplicándolos en forma alternada (sistémicocontacto-sistémico-contacto, etc) si se observa que la enfermedad sigue avanzando. La frecuencia entre aplicación y aplicación será dada por el grado de avance de la enfermedad,

- si la enfermedad no apareciera hasta el inicio de la floración, empezar las aplicaciones con un fungicida de contacto y continuar aplicando sólo fungicidas de contacto,

- la última aplicación no debe realizarse con un fungicida sistémico,

- los cultivares con los cuales se ha desarrollado esta estrategia tienen una resistencia proporcionada por genes $\mathrm{R}$ vencidos (Rd), posiblemente en combinación con genes menores para resistencia horizontal (Fernández-Northcote y Plata, 1998; Navia y Fernández-Northcote, 1996a).

Como se observa en las estrategias para cultivares resistentes, la primera aplicación de fungicida se realiza al inicio de la enfermedad (primeros síntomas). En cambio, en cultivares susceptibles es muy importante que la primera aplicación de fungicida se realice en forma preventiva, antes de que aparezcan los primeros síntomas del tizón.

Al presente, debido a los escasos recursos económicos del agricultor no se sugiere el uso de dos o más fungicidas sistémicos. Sin embargo, conforme un mayor número de agricultores en una zona determinada practiquen las estrategias, será conveniente la diversificación usando por lo menos dos sistémicos de grupos diferentes por campaña. En este sentido, lo ideal sería utilizar una fenilamida o fosetil-al alternados con un sistémico de otro grupo, cuando la estrategia indique el turno para el sistémico. 


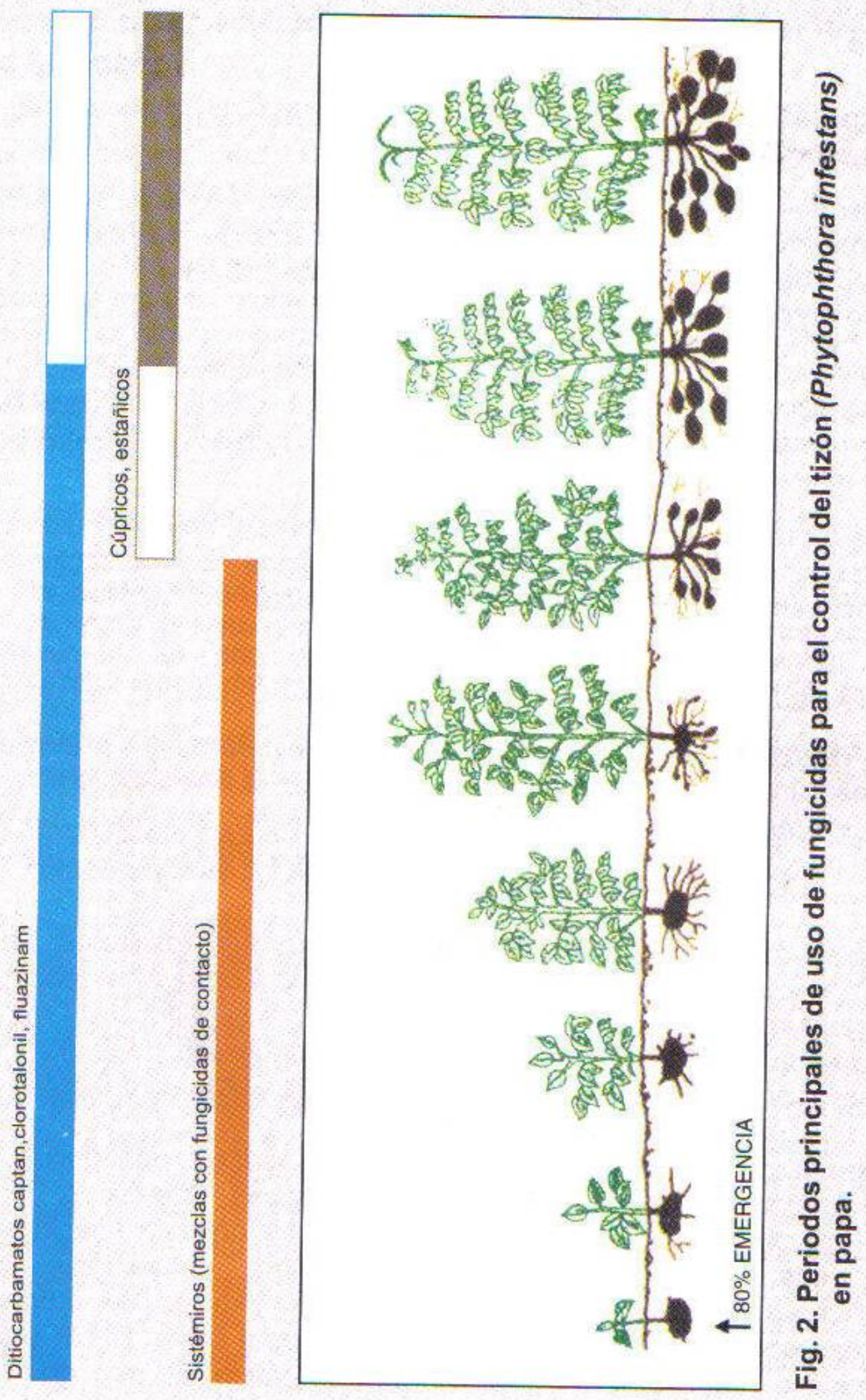




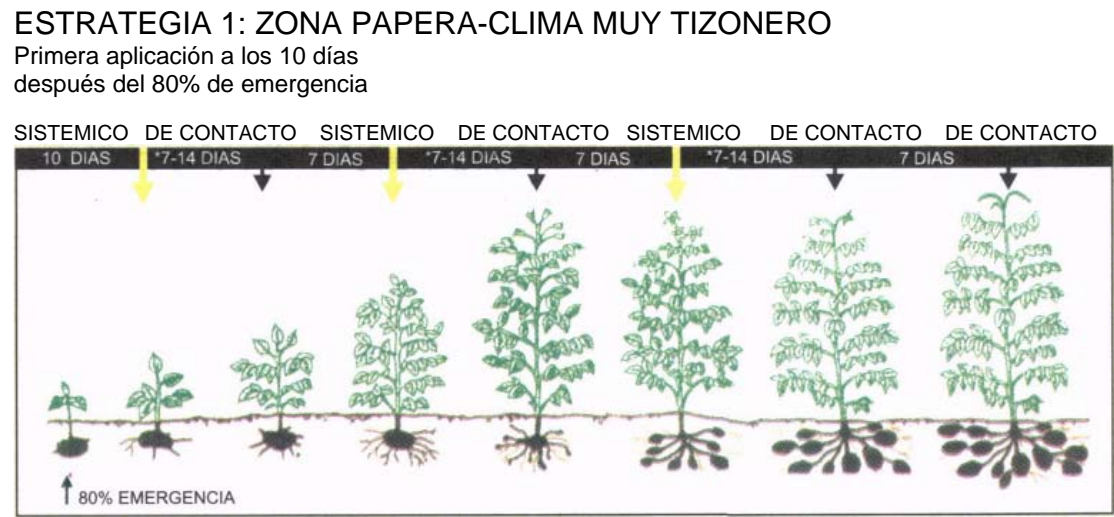

ESTRATEGIA 2: ZONA PAPERA-CLIMA NO MUY TIZONERO

Primera aplicación a los 10 días después del $80 \%$ de emergencia

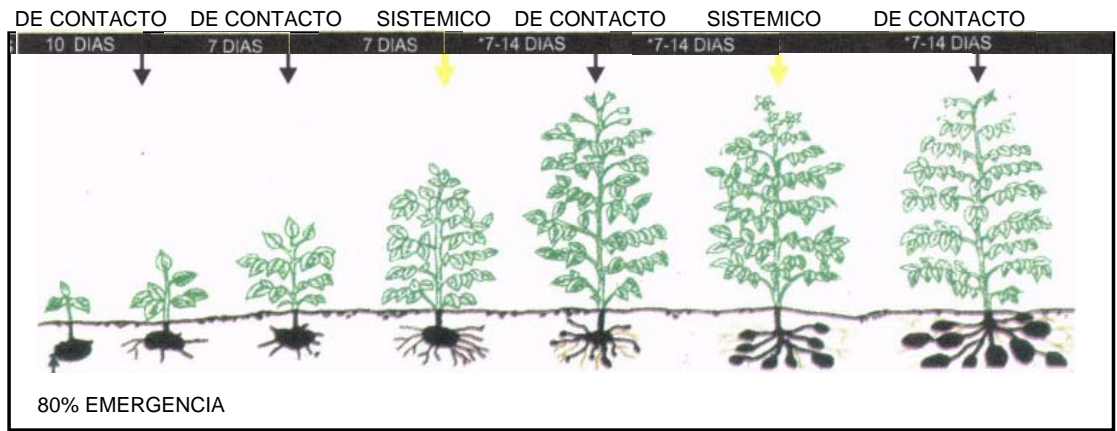

Fig. 3. Estrategias de control químico del tizón (Phytophthora infestans) para cultivares de papa susceptibles.

$\begin{array}{ll}\begin{array}{l}\text { Primera aplicación al inicio } \\ \text { de la enfermedad }\end{array} & \begin{array}{l}\text { Segunda aplicación según el } \\ \text { avance de la enfermedad }\end{array}\end{array}$

SISTEMICO DE CONTACTO

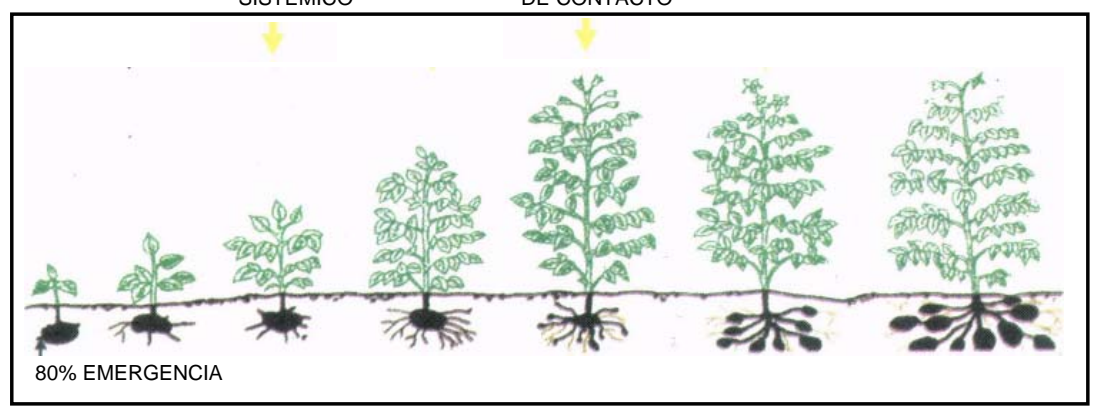

*Alta humedad o precipitación: Aplicar a los 7 días.

Baja humedad o precipitación: Aplicar a los 14 días.

Fig. 4. Estrategia de control químico del tizón (Phytophthora infestans) para cultivares de papa resistentes. 
Las aplicaciones de fungicidas se deben realizar usando las dosis recomendadas comercialmente, con una boquilla apropiada que produzca gotas finas y rociando bien la planta, especialmente la cara inferior de los foliolos (envés) en el caso de los fungicidas de contacto. Se debe evitar que el producto escurra al suelo a fin de ahorrar en el costo de aplicación y evitar la contaminación del medio ambiente.

Ante la admiración de los agricultores la utilización de estas estrategias está permitiendo que se utilicen cultivares susceptibles en zonas y épocas donde ya no se podía cultivarlos por la alta incidencia del tizón, así como interesarse nuevamente en cultivares que en estas mismas zonas consideraban que habían perdido resistencia.

Bajo condiciones muy tizoneras, para poder lograr una buena producción normalmente los agricultores necesitan realizar entre 11 y 16 aplicaciones. Con la Estrategia 1 se requiere alrededor de seis aplicaciones para un buen control de la enfermedad, es decir que hay por lo menos un $50 \%$ de reducción en la cantidad de fungicidas a utilizarse. Es común que en estas zonas el agricultor que no conoce estas estrategias realice solo dos a cuatro aplicaciones debido a sus escasos recursos económicos y en momento inoportuno, como consecuencia pierde su cultivo u obtiene muy bajos rendimientos. Si las aplicaciones las realiza el agricultor siguiendo la Estrategia 1 aún con las dos primeras aplicaciones se obtiene un rendimiento generalmente de más del doble de lo que obtendría el agricultor con» su modalidad de aplicación, y a mayor número de aplicaciones mayor producción (Fernández-Northcote y Navia, 1995; Navia et al., 1996). Un análisis de la rentabilidad de la estrategia para el control químico del tizón en cultivares susceptibles en el área de Morochata, Cochabamba, mostró que durante cinco años de ensayos el promedio del costo marginal de usar la estrategia frente a la práctica del agricultor fue de alrededor de \$ US 100 por ha, los beneficios marginales fueron de casi \$ US 1,400 por ha y la tasa de retorno marginal mayor al 1,300\%. La estrategia sigue siendo muy rentable aún cuando el precio de la papa baje o los costos del control químico suban (Thiele et al., 1998).

Con la estrategia para cultivares resistentes se requieren entre dos a cuatro aplicaciones en una zona muy tizonera, mientras que bajo las mismas condiciones en un cultivar susceptible se requerirían entre 6 a 8 aplicaciones para lograr un buen control del tizón con la estrategia para cultivares susceptibles (Estrategia 1). Es decir, se logra por lo menos un $50 \%$ de reducción en la cantidad de fungicida por ha. El costo del tratamiento químico en los cultivares resistentes representa generalmente no más del $30 \%$ del costo para un buen control de la 
enfermedad en el cultivar susceptible, que por otro lado tienen un rendimiento inferior al obtenido con los cultivares resistentes (Navia y Fernández-Northcote, 1996a).

\section{Ventajas de las estrategias de PROINPA:}

Entre las ventajas más destacables de las estrategias para el control químico del tizón se mencionan:

- logran también el control de la mancha foliar por Alternaria solani

- coinciden con las recomendaciones del comité internacional antiresistencia para sistémicos(Nuninger et al., 1995)

- toman en cuenta la idiosincrasia del agricultor ya que la utilización de fungicidas sistémicos brinda una mayor garantía de protección, facilitándole la movilización para atender sus otras parcelas generalmente ubicadas en zonas distantes, así como otras actividades. Por otro lado los fungicidas sistémicos aminoran las deficiencias en las aplicaciones de otros fungicidas

- protegen la salud del agricultor al reducirse el número de aplicaciones y evitan el uso excesivo de ditiocarbamatos

- protegen el medio ambiente al reducirse la cantidad de fungicidas necesarios por lo menos a un 50\% y promover un uso racional de los fungicidas,

- contribuyen a la superación socio-económica del agricultor.

\section{Innovaciones en las estrategias:}

La implementación de innovaciones debe ser algo dinámico, constantemente aparecen en el mercado nuevos productos que es necesario evaluar y determinar su incorporación en las estrategias.

Al presente se viene evaluando el uso de activadores de resistencia (Trujillo et al., 1997; Carrasco et al., 1998). Metalaxil-M es un nuevo producto que ha sido evaluado e incorporado en las estrategias de PROINPA (Carrasco et al., 1998). Este producto contiene el enantiómero (R-enantiómero) más activo de los dos enantiómeros del fungicida metalaxil (R-y L- enantiómeros). El Metalaxil-M contiene la primera forma enantiomérica de un fungicida introducida al mercado. Se utiliza a la mitad de la concentración que se utiliza para el metalaxil común reduciéndose por lo tanto la cantidad de fungicida necesaria por hectárea. Tiene además una degradación en el suelo más rápida (Nuninger et al., 1996). También se están evaluando productos más específicos para un mejor control de la mancha foliar por Alternaría, como 
parte de las estrategias.

Para las zonas no muy tizoneras se está estudiando un sistema de predicción del tizón que permita superar la eficiencia de la estrategia para estas zonas.

A fin de reducir a! máximo el uso de fungicidas, PROINPA está incentivando el uso de cultivares resistentes bajo la estrategia de control químico de PROINPA para estos cultivares. Se está evaluando también el efecto de esta integración en el control químico de los cultivares susceptibles cuando estos están intercalados con los cultivares resistentes (Navia et al.,1998).

\section{Transferencia de las estrategias:}

Para una transferencia apropiada de las estrategias, es importante considerar que el tizón de la papa es un tipo de enfermedad equivalente en la actualidad al SIDA, la tuberculosis, brucelosis, o cólera, que afectan a los humanos, para cuya prevención o tratamiento apropiado sin consecuencias dañinas posteriores hay medidas que deben necesariamente cumplirse y que actualmente necesariamente tienen que hacerse.

La ambientación político-económica facilitaría la transferencia de las estrategias, así el agricultor tomaría más conciencia sobre la importancia de salir de una agricultura de subsistencia a una agricultura de mayor producción y productividad para los próximos 20 años y quien no controle eficientemente al tizón no sólo se afecta a sí mismo sino que afecta a sus vecinos y a su comunidad, y pierde por otro lado la oportunidad de mejorar su condición socioeconómica.

PROINPA se encuentra al presente en un proceso activo de transferencia de las estrategias contando con la colaboración de firmas representantes de los fungicidas que se comercializan en el país (Villaroel et al, 1996; Salazar et al., 1996; Velazco et al., 1997; Equize y Ortega, 1998; Macías, 1998; Veizaga, 1999). Se están implementando escuelas de campo para la capacitación del agricultor en el tizón, las estrategias de PROINPA para su control químico, y manejo integrado (Torrez y Thiele, 1998). 


\section{Referencias Bibliográficas}

1. Bojanic, A. 1995. Sondeo sobre demanda nacional de semilla de papa para el sector formal y su pertinencia para la UPS-SEPA. Informe.Cooperación Técnica Suiza. La Paz, Bolivia.

2. Bruck, R.I; Fry, W.E.; Apple, A.E. 1980. Effect of metalaxyl, an acylanine fungicide on developmental stages of Phytophthora infestans. Phytopathology 70: 597-601.

3. Carrasco, R.; Navia, O.; Gandarillas, A. 1998. Estrategias del control químico del tizón en cultivares susceptibles. Utilización de un activador de resistencia. En: PROINPA Informe Anual 19971998. Cochabamba, Bolivia. p. 261-264.

4. Cole, D.; Carpio, F.; Julian, J.; Math, M.; Leon, N. 1997. Dermatitis in Ecuadorean farm workers. Contact Dermatitis 37:1-8.

5. Crissman, C. 1994. Pesticide use and farm worker health in Ecuadorean potato production. Amer. J. Agr. Econ. 76: 593-597.

6. Davidse, L,C.; Looijen, D.; Turkensteen, L.J. ; van der. Wal, D. 1981. Ocurrence of metalaxyl-resistant strains of Phytophthora infestans in Dutch potato fields. Netherlands Journal of Plant Pathology 87:65-68.

7. Dowley, L.J.; Cooke, L.R; O'Sullivan, E. 1995. Development and monitoring of an anti-resistance strategy for phenylamide use against Phytophthora infestans. Phytophthora infestans 150. L.J. Dowley, E. Bannon, L.R. Cooke, T. Keane, E. O' Sullivan (eds.). European Association for Potato Research-Pathology Section Conference, Dublin, Ireland, September 1995. Boole Press Ltd.

8. Dowley, L.J.; O' Sullivan, E. 1981. Metalaxyl-resistant strains of Phytophthora infestans (Mont.) de Bary in Ireland. Potato Research 24:417-421.

9. Emmerman, A. 1996. Sweden's reduced risk pesticide policy. Pesticides News No. 34 : 6.

10. Equize, H.; Ortega, D. 1998. Validación de la estrategia de control químico del tizón (Phytophthora infestans) a nivel de agricultor en Chuquisaca, Bolivia. En: Compendio de Exposiciones XVIII Reunión de la Asociación Latinoamericana de la Papa. Cochabamba, Bolivia. Febrero 9-13, 1998. p. 85-86.

11. Evenhuis, A.; Schepers, H.T.A.M.; Bus, C.B.; Stegeman, W. 1996. 
Synergy of cymoxanil and mancozeb when used to control potato late blight. Potato Research 39: 551-559.

12. Fernández-Northcote, E.N.; Navia, 0.1995. Manejo integrado del tizón (MIP-TIZON). En: Manejo integrado de plagas de papa. Memorias del II Curso Internacional de Manejo Integrado de Plagas de Papa. Curso de Capacitación a Distancia. Marzo 20-25,1995. p. 130-136.

13. Fernández-Northcote, E.N.; Plata, G. 1998. Bases para una resistencia duradera al tizón mediante la utilización de cultivares de papa con genes-R vencidos dentro de un manejo integrado. En: Compendio de Exposiciones de la XVIII Reunión de la Asociación Latinoamericana de la Papa. Cochabamba, Bolivia. Febrero 9-13, 1998. p. 83-84.

14. Flier, W.G.; Turkensteen, L.J.; van den Bosh, G.B.M. 1998. Incremento de la agresividad y variación genética en las poblaciones sexuales de Phytophthora infestans en Holanda. En: Compendio de Exposiciones XVIII Reunión de la Asociación Latinoamericana de la Papa. Cochabamba, Bolivia. Febrero 9-13, 1998. p. 255-256.

15. Fry, W.E.; Goodwin, S.B.; Dyer, A.T.; Matuszak, J.M.; Drenth, A.; Tooley, P.W.; Sujkowski, L.S.; Koh, Y.J.; Cohen, B.A.; Spielman, L.J.; Deahl, K.L.; Inglis, D.A.; Sandlan, K.P. 1993. Historical and recent migrations of Phytophthora infestans: chronology, pathways, and implications. Plant Disease 77:653-661.

16. Guo, Z.; Miyoshi, H.; Komoyoji, T.; Haga, T.; Fujita, T. 1990. Uncoupling activity of a newly developed fungicide, Fluazinam. Biochemica et Biophysica Acta 1056: 89-92.

17. Horton, D. 1992. La Papa. Producción, Comercialización y Programas. CIP, Lima y Hemisferio Sur, Montevideo.

18. ISK- Biotech. 1990. Bravo, Daconil 12787. Broad spectrum fungicide. ISK- Biotech Corporation. Ohio, USA.

19. Jorgensen, L.N. 1996. Measures to cut Danish fungicide use. Pesticides News No. 34:7.

20. Leroux, P; Chabane, K.; Bompeix, G. 1993. Selection and characterization of Phytophthora parasítica mutants with ultravioletinduced resistance to dimethomorph or metalaxyl. Pesticide Science 39: 325-329.

21. Macías, E. 1998. Validación participativa de la estrategia de control químico del tizón (Phytophthora infestans) con agricultores en las comunidades de Morochata. Tesis Ing. Agr. UMSS, Cochababmba, Bolivia.

22. Navia, O.; Fernández-Northcote, E.N. 1996a. Estrategias para la integración de resistencia y control químico del tizón. Fitopatología, 
Ficha Técnica 3, PROINPA. Cochabamba, Bolivia.

23. Navia, O.; Fernández-Northcote, E.N. 1996b. Manejo integrado del tizón (MIP-Tizón). Fitopatología, Ficha Técnica 4, PROINPA. Cochabamba, Bolivia.

24. Navia, O.; Equize, H.; Fernández-Northcote, E.N. 1996. Estrategias para el control químico de tizón. Fitopatología, Ficha Técnica 2, PROINPA. Cochabamba, Bolivia.

25. Navia, O.; Gandarillas, A..; Fernández-Northcote, E.N. 1998. Integración de resistencia con control químico del tizón. Efecto de cultivares resistentes en el control químico del tizón en un cultivar susceptible. En: PROINPA Informe Anual 1997-1998. Cochabamba, Bolivia, p. 265-268.

26. Navia, O.; Fernández-Northcote, E.N. 1999. Manejo integrado del tizón. En: Memorias del Taller sobre Manejo Integrado del Tizón Tardío de la Papa en la Ecoregión Andina. Quito, Ecuador. Abril 7-9, 1997. p. 1-12.

27. Nuninger, C.; Steden, C.; Staub, T. 1995. The contribution of metalaxyl-based fungicide mixtures to potato late blight control. Phytophthora infestans 150. L.J. Dowley, E. Bannon, L.R. Cooke, T. Keane, and E. O'Sullivan (eds.). European Association for Potato Research-Pathology Section Conference. Dublin, Ireland, September 1995. Boole Press Ltd.

28. Nuninger, C.; Watson, G.; Leadbitter, N.; Ellgehausen, H. 1996. CGA 329351: Introduction of the enantiomeric form of the fungicide metalaxyl. En: Ciba's Contribution. Paper presented at the Brighton Crop Protection Conference-Pest and Diseases, 1996. Ciba-Geigy Ltd. Basle, Switzerland. p. 30-35.

29. Papavizas, G.C.; O'Neill, N.R.; Lewis, J.A. 1978. Fungistatic activity of propyl-N-(alpha-dimethylaminopropyl) carbamate of Pythium spp. and its reversal by sterols. Phytopathology 68: 1667-1671.

30. Rojas, L. .1995. Selección de estrategias de control químico del tizón (Phytophthora infestans) en cultivares de papa susceptibles y resistentes en Cochabamba. Tesis Ing. Agr. UMSS, Cochabamba, Bolivia.

31. Salazar, M. 1996. Evaluación inicial del grado de adopción de la estrategia para el control químico del tizón [Phytophthora infestans (Mont.) de Bary] en seis comunidades de Morochata. Tesis Ing. Agr. UMSS, Cochabamba, Bolivia.

32. Salazar, M.; Thiele, G.; Tórrez, R. 1996. Evaluación inicial de adopción de la estrategia de control químico del tizón (Phytophthora infestans) en Morochata. En: Compendio de Exposiciones IV Reunión Nacional de la Papa. Cochabamba, Bolivia. Octubre 8-11,1996. p. 13-14. 
33. Samoucha, Y.; Cohen, Y. 1989. Field control of potato late blight by synergistic fungicidal mixtures. Plant Dis. 73: 751-753.

34. Samoucha, Y.; Cohen, Y. 1990. Toxicity of propamocarb to the late blight fungus on potato. Phytoparasitica 18 (1): 27-40.

35. Schwinn, FJ.; Margot, P,. 1991. Control with chemicals. En: Phytophthora infestans, the cause of late blight of potato. Advances in Plant Pathology. D.S. Ingram and P.H. Williams, (eds.), Academic Press, London. p. 225-265.

36. The Pesticides Trust. 1994. The List of Lists. The Pesticides Trust. London, UK.

37. Thiele, G.; Navia, O.; Fernández-Northcote, E.N. 1998. Análisis económico de la estrategia de control químico del tizón (Phytophthora infestans) para cultivares de papa susceptibles en Cochabamba, Bolivia. Fitopatología 33(3):176-181.

38. Torrez, R.; Thiele, G. 1998. El uso de jampis, épocas de siembra y cultivares: diagnósticos participativos y capacitación en el manejo integrado del tizón. En: Compendio de Exposiciones XVIII Reunión de la Asociación Latinoamericana de la Papa. Cochabamba, Bolivia. Febrero 9-13, 1998. p. 190-191.

39. Trujillo, A.; Navia, O.; Fernández-Northcote, E.N. 1997. Integration of resistance and chemical control for late blight. Use of a resistance activator. En: Libro de Resúmenes, IX Congreso Latinoamericano de Fitopatología. Octubre 12-17, 1997, Montevideo, Uruguay, p. 254.

40. Velasco, W.; Navia, O.; Fernández-Northcote, E.N. 1996. Oportunidad de aplicación y tipo de fungicidas en la integración de resistencia con control químico para el tizón de la papa. En: Compendio de Exposiciones IV Reunión Nacional de la Papa. Octubre 8-11, 1996. Cochabamba, Bolivia, p. 95-96.

41. Velasco, W.; Trujillo, A.; Navia, O.; Fernández-Northcote, E.N. 1997. Red de ensayos de control químico del tizón de la papa (Phytophthora infestans). Informes de actividades en los módulos de Morochata y Colomi. Campaña 1996-1997. PROINPA, Cochabamba, Bolivia.

42. Villaroel, J.; Navia, O.; Fernández-Northcote, E.N. 1996. Red de ensayos de validación de estrategias de control químico del tizón para cultivares susceptibles. En: Compendio de Exposiciones IV 
Reunión Nacional de la Papa. Octubre 8-11, 1996. Cochabamba, Bolivia, p. 173.

43. Veizaga, A. 1999. Evaluación de la capacitación en la estrategia de control químico del tizón (Phytophthora infestans), en la zona de Morochata. Tesis Ing. Agr. UMSS, Cochabamba, Bolivia.

44. Wade, M; Delp, C.J. 1985. Aims and activities of industry's fungicide resistance action committee (FRAC). EPPO Bulletin 15: 577-583.

45. Williams, R.J.; Gisi, U. 1992. Monitoring pathogen sensitivity to phenylamide fungicides: principies and interpretation. EPPO Bulletin 22:297-322.

46. Zeballos, H. 1997. Aspectos económicos de la producción de papa en Bolivia. COSUDE-Centro Internacional de la Papa(CIP).Lima, Perú.

47. Ziogas, B.N.; Davidse, L.C. 1987. Studies on the mechanism of action of cymoxanil in Phytophthora infestans. Pestic. Biochem. Physiol. 29:89-96. 